\title{
Verification of the virtual bandwidth SAR (VB-SAR) scheme for centimetric resolution subsurface imaging from space
}

Article

Accepted Version

Edwards-Smith, A., Morrison, K., Zwieback, S. and Hajnsek, I. (2018) Verification of the virtual bandwidth SAR (VB-SAR) scheme for centimetric resolution subsurface imaging from space. Transactions on Geoscience and Remote Sensing, 56 (1). pp. 25-34. ISSN 0196-2892 doi:

https://doi.org/10.1109/TGRS.2017.2717340 Available at https://centaur.reading.ac.uk/73209/

It is advisable to refer to the publisher's version if you intend to cite from the work. See Guidance on citing.

To link to this article DOI: http://dx.doi.org/10.1109/TGRS.2017.2717340

Publisher: IEEE

All outputs in CentAUR are protected by Intellectual Property Rights law, including copyright law. Copyright and IPR is retained by the creators or other copyright holders. Terms and conditions for use of this material are defined in the End User Agreement. 


\section{CentAUR}

Central Archive at the University of Reading

Reading's research outputs online 


\title{
Verification of the Virtual Bandwidth SAR (VB-SAR) Scheme for Centimetric Resolution Subsurface Imaging From Space
}

\author{
A. Edwards-Smith, K. Morrison, S. Zwieback, I. Hajnsek
}

\begin{abstract}
This work presents the first experimental demonstration of the virtual bandwidth synthetic aperture radar (VB-SAR) imaging scheme. VB-SAR is a newly-developed subsurface imaging technique which, in stark contrast to traditional close-proximity ground penetrating radar (GPR) schemes, promises imaging from remote standoff platforms such as aircraft and satellites. It specifically exploits the differential interferometric synthetic aperture radar (DInSAR) phase history of a radar wave within a drying soil volume to generate highresolution vertical maps of the scattering through the soil volume. For this study, a stack of C-band VV polarisation DInSAR images of a sandy soil containing a buried target was collected in the laboratory whilst the soil moisture was varied - firstly during controlled water addition, and then during subsequent drying. The wetting image set established the moisture-phase relationship for the soil, which was then applied to the drying DInSAR image set using the VB-SAR scheme. This allowed retrieval of high resolution VB-SAR imagery with a vertical discrimination of $0.04 \mathrm{~m}$ from a stack of $1 \mathrm{~m}$ vertical resolution DInSAR images. This work unequivocally shows that the basic principles of the VB-SAR technique are valid and opens the door to further investigation of this promising technique.
\end{abstract}

Index Terms - ground penetrating radar, radar, radar imaging, spaceborne radar, synthetic aperture radar

\section{INTRODUCTION}

N ubsurface radar imaging is traditionally performed by nadirviewing, ground penetrating radar (GPR) systems that rely on close contact with the ground surface [1]. This limits the areas that can be safely surveyed to areas that are non-hazardous and easily accessible. Even for sites that are easily accessible the relatively slow process of GPR means that surveying large areas is slow, and hence expensive, not to mention difficult [2]. Relatively benign terrain undulations can introduce an additional level of complication to the surveying process, requiring extremely precise measurement of the antenna position during the surveying process [3]. Conversely, radar imaging from standoff distances such as that performed by airborne or spaceborne systems overcomes site access issues and speed of acquisition. However, stand-off systems have major drawbacks when compared to traditional GPR systems. They typically operate over narrow bandwidths (due to regulatory and technical limitations), leading to significantly decreased slant range resolution.

Whereas GPR systems produce depth profiles which provide the ability to unambiguously discriminate between the depths of different returns, synthetic aperture radar (SAR) systems cannot easily discern between surface and subsurface returns. Although subsurface features have previously been reported in SAR imagery [4-7], in these papers there is no presentation of a depth profile; rather identification of subsurface geological-scale features relied upon intelligent supposition, contextual information, and cross-referencing against optical imagery. Arid desert conditions allowed for significant penetration of the radar signal to tens of meters. However, in more temperate climes with more moisture present in the soil, penetration depths (where the signal has fallen to 1/e of its strength at the soil surface) will be drastically lower and on the order of a wavelength [8]. Surveying the current crop of existing (L-, C-, and X-band) and near-future satellites (adding P-band), quickly indicates that their slant range resolutions are significantly poorer than a wavelength. Thus, all returns will anyway appear unresolved within the viewing depth of the radar, such that meaningful subsurface imaging is not possible. It is in the context of these demanding challenges that the virtual bandwidth synthetic aperture radar (VB-SAR) scheme was proposed [9]. It offers the possibility of addressing these problems by offering a radical new scheme which delivers a depth-resolution capability independent of a radar's bandwidth. Based on a soil-radar model, VB-SAR exploits the differing temporal phase behaviours of soil features at different depths associated with changes in soil moisture content (SMC). Using a differential interferometric SAR (DInSAR) image stack, the scheme can isolate returns with a depth resolution which may be far superior to the formal range resolution of the radar. Thus, objects (including both distinct features such as landmines or archaeological remains and geological features such as bedrock) within the penetration depth of the radar system may now be resolved. Although data will very likely not be collected in equal increments of moisture change, for the imagery to be correctly scaled in depth first requires linearization of the moisture changes before application of the VB-SAR scheme. In this study we present experimental results utilizing a buried trihedral to validate the basic principles of the VB-SAR scheme. We derive imagery with depth resolutions far superior to that across the input DInSAR stack. We also discuss its realworld application and consider possible limitations of the scheme.

\section{MEASUREMENTS}

\section{A. Laboratory Details}

The experimental data for this study were collected using an indoor microwave measurement facility. A view of the facility is shown in Figure 1. A linear scanner is centrally 
located down the length of a $4 \mathrm{~m}(\mathrm{l}) \times 1 \mathrm{~m}(\mathrm{w}) \times 0.9 \mathrm{~m}(\mathrm{~h})$ soil trough. The trough is constructed from plywood with no metal fasteners in order to avoid unwanted radar returns. The radio frequency (RF) subsystem consists of an HP 8719ES Vector Network Analyser (VNA), which acts as both the RF source and RF receiver for the system, connected to the antennas by means of flexible coaxial cable runs. Movement is by a computer controlled servomotor which allows accurate mechanical positioning of the antennas along the scanner in order to allow synthetic aperture techniques to be used. The antennas are momentarily static whilst each RF measurement is made. The system can be automatically set to acquire image sequences at precisely timed intervals. This enables unsupervised regular sampling over long periods of time to study the slowly evolving scattering behavior of a scene.

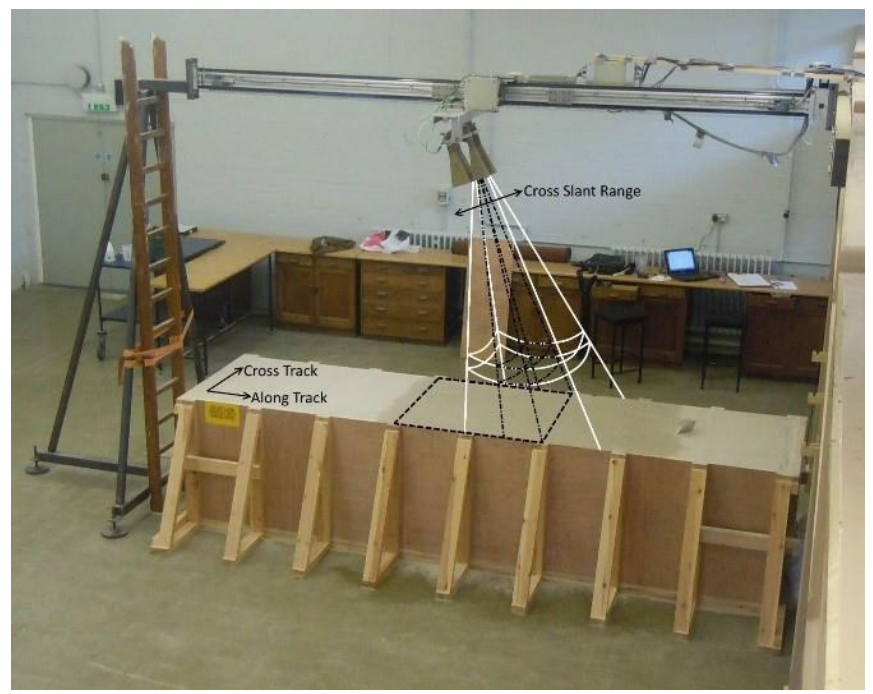

Fig. 1: A view of the soil trough and linear scanner with the C-band antennas attached. Note that this photograph was taken whilst a different experiment was being performed, hence the wetted area and reference trihedral deployment are different to the situation in this study. Dashed square on trough surface shows area actually wetted during this study. Solid white lines represent the real antenna beam pattern and resolution cell, dot-dashed black lines indicate beam pattern and resolution cell obtained using tomographic profiling. Coordinate notation as used in text.

\section{B. Soil Study}

The schematic of the target scene used in this experiment is shown in Figure 2. The trough volume was filled with fine kilndried building sand to act as the soil, except for the central $1 \mathrm{~m}$ x $1 \mathrm{~m}$ region. This region contained a $10 \%$-gravel: $90 \%$-soil mixture by volume, which extended down to a depth of $20 \mathrm{~cm}$. The gravel was randomly shaped and around $1 \mathrm{~cm}$ in diameter. The surface of the sand was smoothed off, level with the trough edges. The gravel-soil mixture sat directly above a square $12 \mathrm{~cm}$ trihedral with a theoretical Radar Cross Section (RCS) of $2.2 \mathrm{~m}^{2}$ at $5 \mathrm{GHz}$. It was tilted backwards to present a maximum RCS at a free space incidence angle of $20^{\circ}$. The apex of the trihedral was placed at a depth of $26.5 \mathrm{~cm}$. Several surface-mounted reference trihedrals were placed at various positions on the soil and trough. Figure 3 illustrates the preparation of the soil sample, showing the gravel-soil mixture placed over the buried trihedral, the wetting of the mixture and the positioning of the surface reference targets.

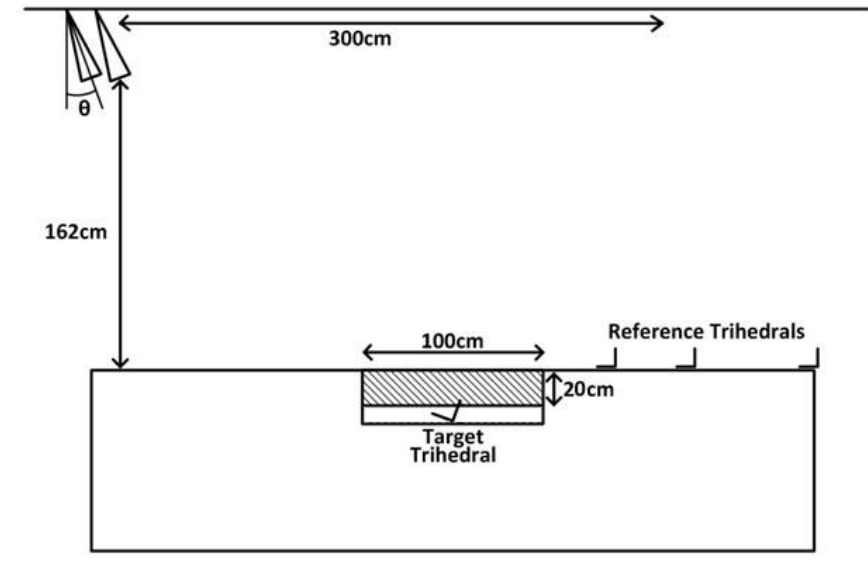

Fig. 2: Schematic of the experimental set-up. The hatched area shows the position of the gravel-soil mixture. The blank area below the hatched area indicates the assumed furthest extent of the added moisture. The apex of the target trihedral is $26.5 \mathrm{~cm}$ below the trough surface.

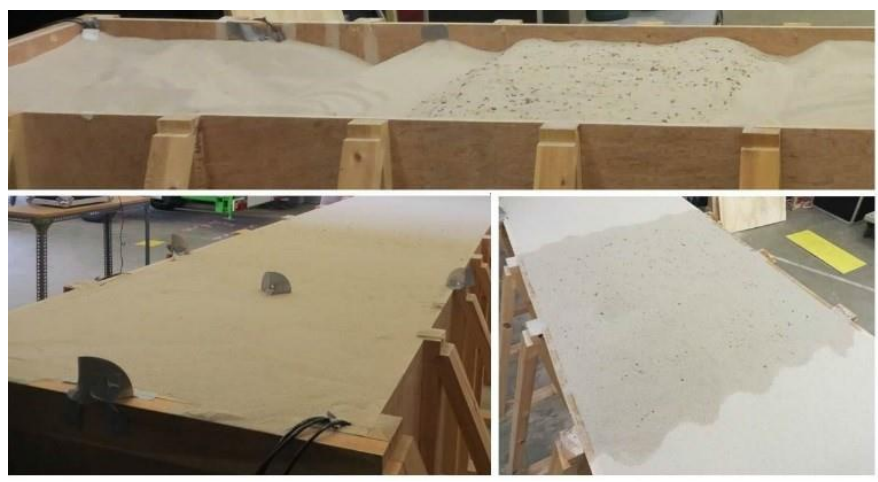

Fig. 3: Top- Preparation of the soil target. Lower right- The wetted $1 \mathrm{~m} \times 1 \mathrm{~m}$ area at the location of the gravel-soil mixture. Lower left- The positioning of the reference trihedrals. Darkening of soil surface in vicinity of surface reference trihedrals is caused by shadow.

The first stage of the experiment involved adding carefully controlled amounts of water to the dry soil and gravel volume above the buried trihedral. This allowed the phase response of the trihedral to changes in soil moisture content to be characterized. The total amount of water added was 26 liters; initially in steps of $200 \mathrm{ml}$ up to 2 liters and then in steps of 2 liters up to 26 liters, added in total. Moisture additions were equally spaced at half hour intervals. The water was added by using a trigger sprayer for the $200 \mathrm{ml}$ additions, and then a watering can with rose was used for the 2 liter additions. A volume of 26 liters was chosen as previous experience indicated this amount of water would cause moisture to travel to down to a depth of $26.5 \mathrm{~cm}$, which informed on the depth placement of the trihedral. Additionally, 26 liters was estimated to represent the point at which attenuation would cause the trihedral to just be lost in the radar imagery, thus providing a maximum variation in signal as the trihedral feature reappeared during the drying phase. Assuming all the water stayed within an $1 \mathrm{~m} \times 1 \mathrm{~m}$ $x 0.265 \mathrm{~m}$ active wet region bounded by the soil surface and the trihedral apex, leads to a final maximum volumetric SMC of $9.8 \%$. Whilst the wetting study was done over one day, the drying study commenced after the final water addition and extended over a much longer 35-day interval. During this time, the scene was reimaged at approximately 80 minute intervals. In order to promote evaporation, two portable fans were used to blow air over the surface of the soil during most daytime 
intervals, positioned so that they were well outside the antenna beam patterns and the airflow did not disturb the surface.

\section{Radar Imaging}

Radar imagery in this study was generated using the tomographic profiling (TP) process [10]. TP does not provide a tomographic scene reconstruction per se, however, the presented result has similarity to the final image product from tomographic schemes, namely a 2D vertical backscattering profile through a volume.

The TP process requires data collected in a similar fashion to conventional SAR imaging across a synthetic aperture; the difference is that for TP the antennas are rotated $90^{\circ}$ so they look along the direction of platform travel and so only image a transect directly below the scanner, rather than out to the side. As such, with TP we sacrifice information in the across-track direction, to importantly gain information in the vertical direction. This is important, and it allows us to spatially isolate surface and subsurface features, and visualize spatially the dynamic processes that occur within a soil that are normally hidden from us in SAR imagery. Figure 4 shows a schematic of the TP process.

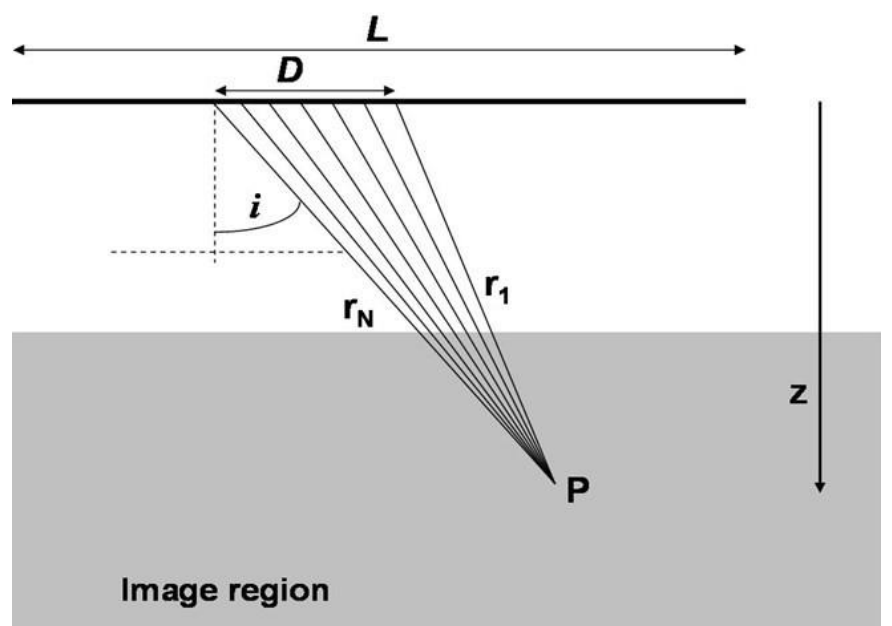

Fig. 4: Schematic of the principle of the TP imaging technique. A scan is made up of a sequence of elemental measurements across the synthetic aperture, $L$. A sub-aperture, $D$, provides a single profile through the image volume at a series of equally-spaced points along the slant-range direction. By sliding the sub aperture along the full aperture, a continuous series of profiles build up the TP image. Importantly, each image point is reconstructed at the same incidence angle, $i$, as used for the point $P$ at depth $z$.

For this study a cluster of C-band antennas was mounted on the scanner, pointing forward at $20^{\circ}$ from nadir. The antennas had $30^{\circ}$ and $50^{\circ} 3 \mathrm{~dB}$ real beamwidths in the alongand across-track directions, respectively, such that surface and volume returns would suffer significant range layover and offer no vertical discrimination through the soil. To overcome this, in the TP scheme the beam is sharpened across a subaperture of the collected synthetic aperture. It is steered to the desired look angle by applying a suitable phase ramp across the subaperture elements. In the slant range direction, resolution is provided by the frequency bandwidth. In the across-track direction (across the width of the trough), however, the synthetic aperture processing does not sharpen the antenna beam; rather, this is given by the width of the real beam. Each subaperture provides an independent narrow-slice look through the soil at the chosen angle, and their successive along-track offsets builds up an image of the scene below the scanner transect. An important property of TP imagery is that the incidence angle is the same at any pixel throughout the image, here chosen to be $10^{\circ}$. The achieved vertical and horizontal resolutions in a TP image are a combination of contributions from the sharpened beam, bandwidth, and real across-track beam [10]. The look angle was chosen as a compromise between high resolution obtained with a steep angle and representative SAR imaging geometry.

All scans were collected over a $3 \mathrm{~m}$ aperture using 151 aperture points and a sampling interval of $2 \mathrm{~cm}$. At each sample position 1601 frequency points were collected over a $2 \mathrm{GHz}$ bandwidth across a frequency range of $4-6 \mathrm{GHz}$. Each scan took just over 6 minutes to collect. In order to remove positioning drift the system was set to "home" against an end stop microswitch after each scan. Analysis indicates that the residual positioning error is controlled to a fraction of an $\mathrm{mm}$, insignificant compared to the wavelength in use and the large phase shifts observed from the buried target.

Two sets of imagery were produced from the data collection. A high resolution image set was produced utilising the full bandwidth available. Slant range, cross-slant range, and across-track resolutions were estimated to be $7.5 \mathrm{~cm}, 35 \mathrm{~cm}$, and $1.3 \mathrm{~m}$, respectively at the soil surface. This leads to vertical and horizontal resolutions of $14 \mathrm{~cm}$ and $36 \mathrm{~cm}$ respectively, at the soil surface. A second low resolution image set was produced using the same parameters as the high resolution reconstructions, but with a reduced bandwidth of $150 \mathrm{MHz}$ across the frequency range of $4-4.15 \mathrm{GHz}$. This gave a slant range estimate of $1 \mathrm{~m}$, leading to a free space vertical resolution of $105 \mathrm{~cm}$ and a horizontal resolution of $52 \mathrm{~cm}$ at the soil surface. Figure 5 shows an example high-resolution TP image (produced from the last scan from the drying period). The buried trihedral is clearly visible in the image. The returns on the right side of the image are two surface reference trihedrals, as well as a group of subsurface features not associated with this study.

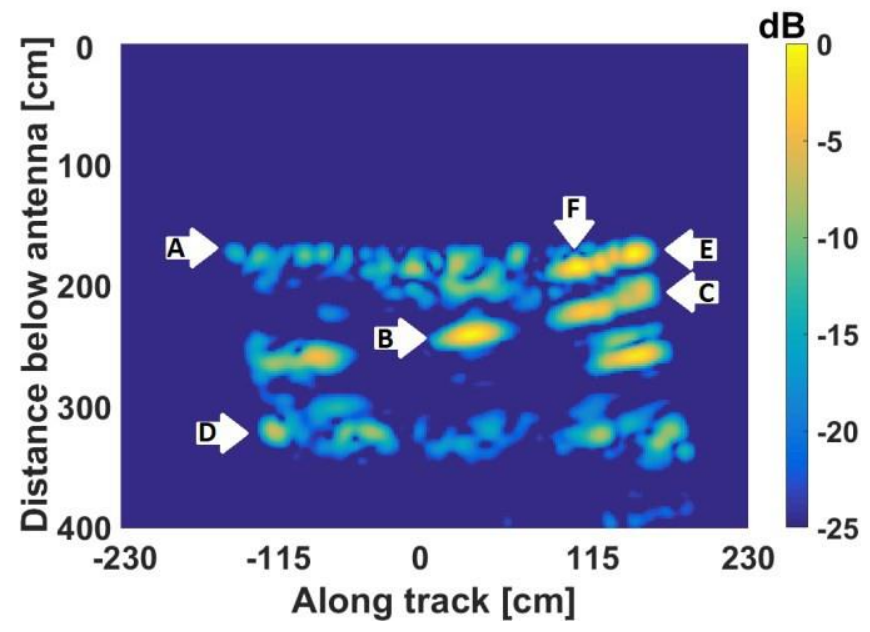

Fig. 5: Example high range resolution TP image prepared using entire real bandwidth. Arrow A indicates the soil surface, with the strong returns at right being generated by some of the surface reference trihedrals, B shows the buried trihedral of interest to this study, C shows returns caused by soil moisture probes present in the sand trough, D shows the returns from the floor of the trough (note attenuated returns in middle of floor, caused by the wetted soil in the region above the trihedral), E shows the return from the single surface reference trihedral in the middle of the trough and $\mathrm{F}$ shows the combined return from the reference trihedrals placed at the edge of the trough (these appear 
below the surface as they were placed on the edges of the trough and the TP process provides no resolution in the cross track direction and so places them onto the central plane of the image).

\section{DATA ANALYSIS}

The analysis strategy is to first use the high-resolution imagery to investigate the "best-performance" result for VBSAR. The resolution allows us to separate the returns from the surface, soil volume, and buried feature. This scheme is then applied to the low resolution imagery in which the returns are no longer separable by depth, and which better represents a realworld SAR application.

The temporal amplitude-phase behavior of the buried trihedral is extracted, first in the wetting period to establish a moisture-phase relationship, and secondly in the drying period for exploitation in a demonstration of the VB-SAR scheme. Behavior is compared with the returns from the surface in the wetted area and from a sub-surface feature within a dry region.

\section{A. Moisture-Phase Relation}

The phase history during the wetting period is of key interest to establish the moisture-phase relationship for the soil, to be utilized in the analysis of the drying period data. A key assumption of VB-SAR is that the variation in phase of a buried target is linear with SMC change [11]. Figure 6 plots the phase response of the buried trihedral to the 2 liter additions of water. As can be seen, the phase decreases linearly with SMC. The phase value extracted from the 26 liter scan is not shown as the buried trihedral was no longer visible due to strong soil attenuation. From Figure 6 we derive a moisture-phase relationship, $\gamma$, of $0.057^{\circ} / \mathrm{ml}$.

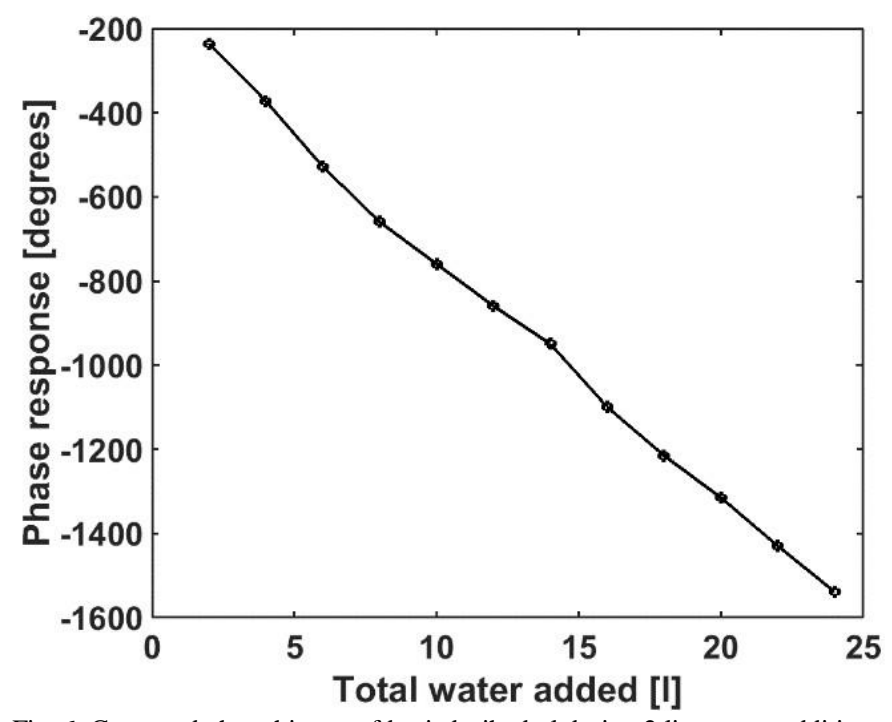

Fig. 6: Corrected phase history of buried trihedral during 2 liter water additions. Trend not plotted after 24 liters added due to soil attenuation obscuring the trihedral.

For comparison, the backscatter history of the buried trihedral is shown in Figure 7. This can be expected to be linear in $\mathrm{dB}$ [11]. Whilst this is mostly the case, there are significantly more fluctuations away from linear than for the phase curve. This supports the ide a that the phase is the more robust parameter in the measurement of SMC, a result found in [11].

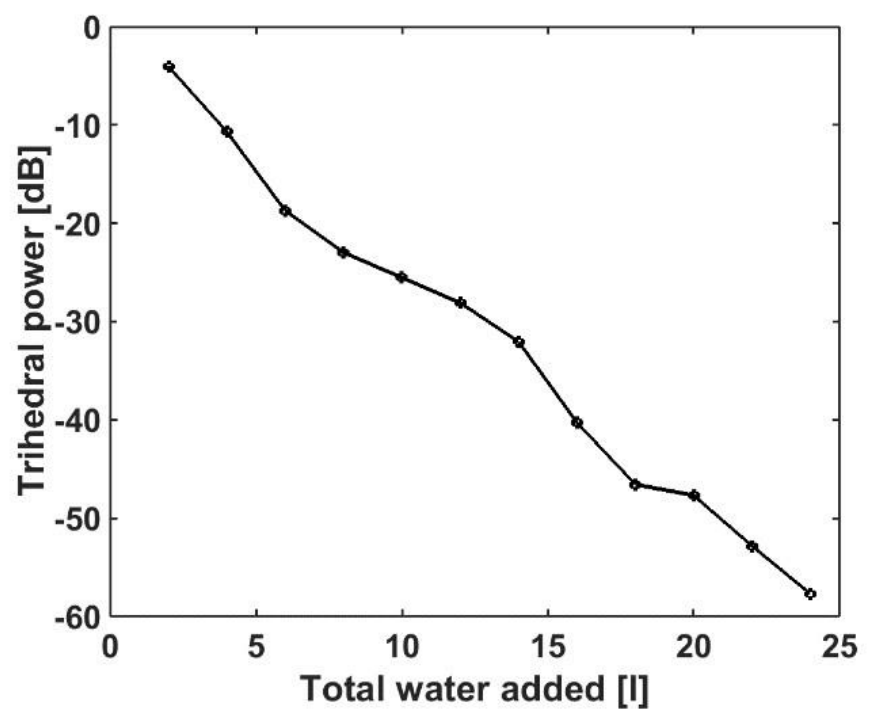

Fig. 7: Backscatter history of buried trihedral during 2 liter water additions. Trend not plotted after 24 liters added due to soil attenuation obscuring the trihedral.

\section{B. Drying Period}

Figure 8 shows a sequence of three TP images over the drying period, from the wettest to driest soil. The buried trihedral was seen to recover in brightness through the interval. Figure 9 and Figure 10 show the phase and backscatter histories, respectively, over the entire drying interval extracted from the pixel corresponding to the buried trihedral from the high resolution TP dataset.

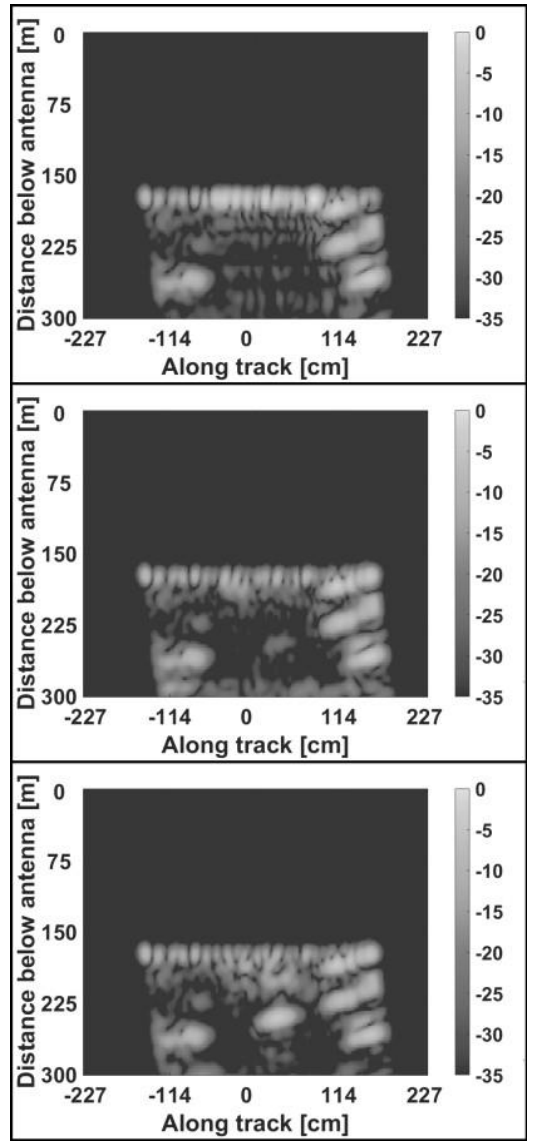


Fig. 8: Selection of TP images from the drying period, with an SMC change of $3.5 \%$ between each image showing the re-emergence of the buried trihedral and the decrease in the surface backscatter as the soil dries. Upper image is the first scan of the drying period (with an SMC of 9.8\%), the middle image is from the middle of the drying period (6.3\% SMC) and the lower image is the last scan collected as part of this study (2.7\% SMC) Presented over a 35dB dynamic range, images normalized relative to each other.

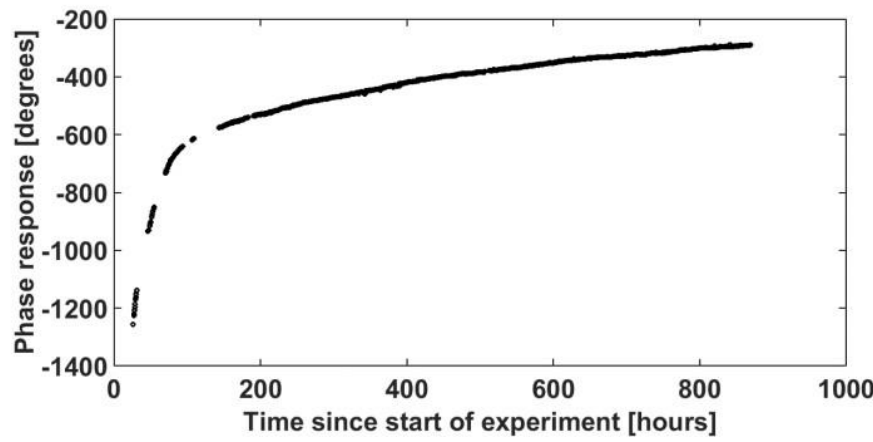

Fig. 9: Phase history for the buried trihedral during drying period after corrections applied

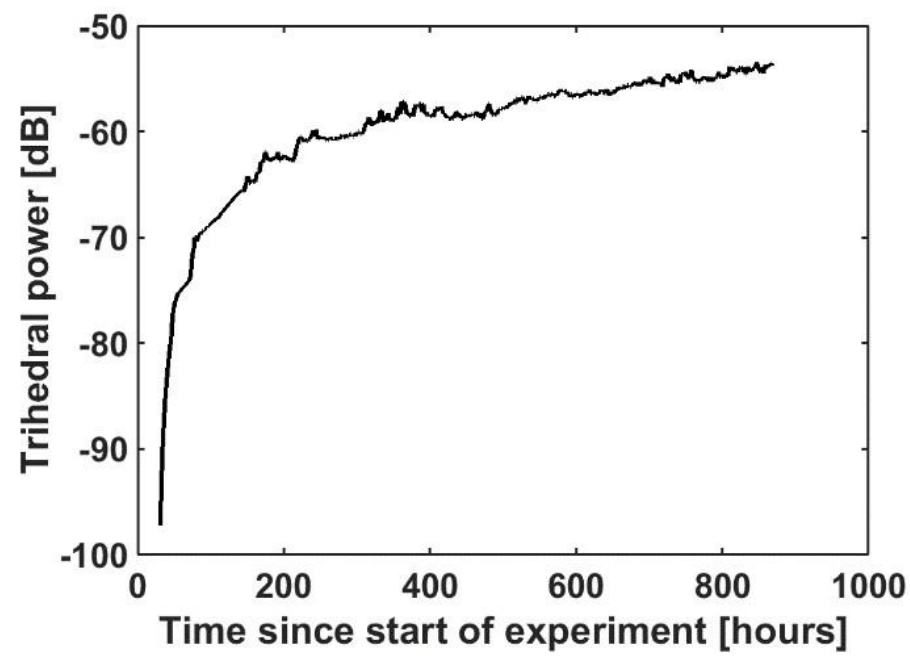

Fig. 10: Backscatter history for the buried trihedral during drying period after corrections applied

The curves have been corrected for any unwanted artefacts of the measurement system by referencing them against the primary reference trihedral on the soil surface. This corrected for system drift which is principally due to diurnal temperature fluctuations in the laboratory.

With the value of $\gamma$ derived above from the wetting period, it was possible to quantitatively interpret the observed phase curve in Figure 9 in terms of SMC. As phase change should be linear with SMC change [9], [11], Figure 9 shows that the change in SMC was very non-linear over the drying period. It is interesting to note that visually two different drying processes appear to be present; a rapid one dominant until the differential phase had returned to -600 degrees after around 150 hours of drying, which then rapidly settled into a much slower drying process until the end of the experiment. We might conjecture that this can be explained by the initial drying process being dominated by the evaporation of moisture from the upper portion of the soil volume, then once the upper portion was dry the loss of moisture was from the lower portions which could reasonably be expected to be a slower process.
With a starting SMC of $9.8 \%$, the total phase change in Figure 9 indicates a drying to $2.7 \%$. These SMC estimates assume that the added water spread homogeneously within the active gravel-sand region and did not move outside this region. Previous work [11] informed on the choice of total water added, which was expected to travel down to just the depth of the trihedral. Previous work has also shown the phase result is little affected by the distribution of SMC above a buried target, only by the total water column above a target [9].

There are small gaps in the measurements in Figure 9, primarily near the start of the data. Because the phase variation is smooth it was meaningful to interpolate across these regions to recover missing data. It was decided to resample the whole data set to 1 hour intervals, slightly higher than the nominal sampling rate of 80 minutes, to ease data manipulation and visualisation.

\section{VB-SAR}

\section{A. VB-SAR Theory}

The dielectric properties of a soil perceived by a radar wave are set by its chemical make-up and water content. Over the timescales of interest for remote sensing, only the moisture can be expected to change so the variations in dielectric properties can be assumed to be solely dependent upon SMC variations [8], [12]. The VB-SAR imaging scheme proposed by [9] leverages upon the fact that the soil dielectric properties over a series of DInSAR acquisitions will vary in a predictable manner quantifiable according to the SMC variations.

The details of the VB-SAR model and technique have previously been described in [9], but are briefly reviewed here. As the refractive index of a soil is higher than the refractive index of air, a radar wave is compressed within the soil. Thus, a given real frequency in air, $f_{R}$, will behave with respect to phase as a wave of a higher virtual frequency, $f_{V}$, within the soil according to;

$$
f_{V}=f_{R} \sqrt{\epsilon_{r}}
$$

where $\sqrt{\epsilon_{r}}$ is the refractive index. This is a central principle of the VB-SAR process. Consider a temporal sequence of DInSAR acquisitions over a drying soil; the changing refractive index of the soil will lead to the fixed real frequency being transported across a range of virtual frequencies. This synthesises a virtual bandwidth, $B_{V}$, over the series of images set by the total change in the refractive index;

$$
B_{V}=f_{R} \Delta \sqrt{\epsilon_{r}}
$$

These virtual frequencies can be treated just as for a real bandwidth.

\section{B. Data Preparation}

Figure 9 indicates that the SMC change was not linear over the image stack during this study. The VB-SAR scheme expects the provision of an interferometric time series at equal intervals of soil moisture change. Thus, in order to prepare the data for use with VB-SAR, the data shown Figure 9 and Figure 10 were resampled to be linear in SMC. Knowing the starting SMC was $9.8 \%$, and using the $\gamma$ term with the Figure 9 phase changes, it was possible to calculate the absolute SMC for each measurement. This was then resampled to provide a linear 
change in SMC as shown in Figure 11.

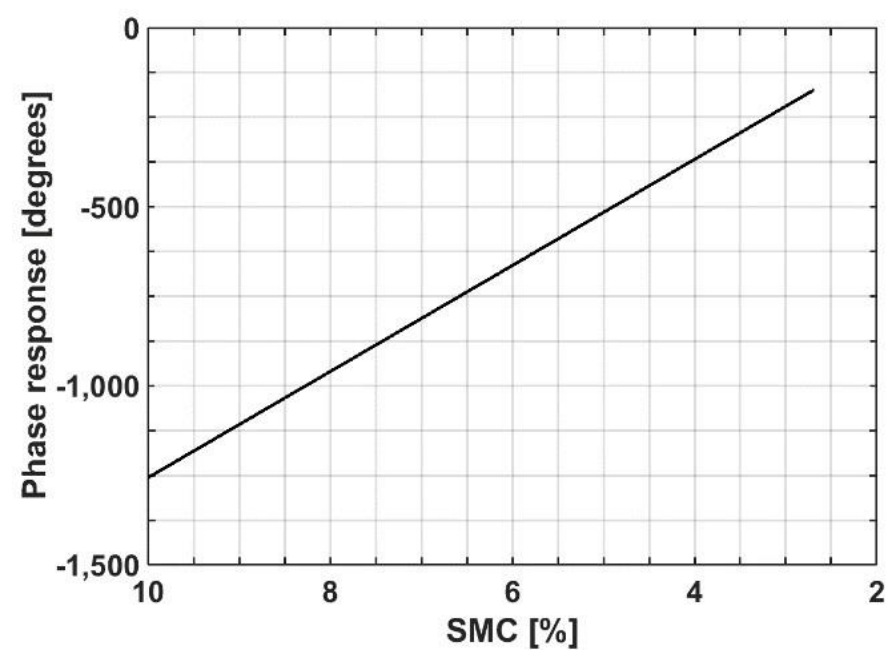

Fig. 11: Phase history of buried trihedral interpolated to linear steps in SMC.

\section{VB-SAR Processing}

The resampled and windowed amplitude and phase histories are then passed through a complex FFT to translate the data from the frequency domain to the time (equivalently, range) domain. A Kaiser window with $\beta$ set to 0.2 was found to give acceptable sidelobe reductions, and allowed sidelobes to be almost eliminated from the final displayed VB-SAR image whilst keeping spreading in the depth dimension to an acceptable level.

The VB-SAR process detailed so far would provide a subsurface depth profile, albeit one without any sense of scale in the depth direction. In order to apply a scale to the depth profile the unambiguous range in the image, $R_{\text {unabg }}$, must be calculated. This is calculated in the usual fashion but using the virtual frequency step size $\left(f_{\text {vstep }}\right)$ and scaling by the average refractive index of the soil across the DInSAR stack, $n_{a v}$, i.e.

$$
R_{\text {unabg }}=\frac{c n_{a v}}{2 f_{v s t e p}}
$$

where $c$ is the speed of light in free space. Note, that in order to calculate $f_{v s t e p}$ and $n_{a v}$ accurately the absolute SMC in each scan must be known. With a known SMC in each scan and a known soil texture the refractive index of the moist soil can be calculated using published soil models [8], [12], from which Equations (2) and (4) can be used to calculate $f_{v s t e p}$;

$$
f_{v s t e p}=\frac{B_{v}}{n_{\text {scans }}}
$$

where $n_{\text {scans }}$ is the number of scans across which the virtual bandwidth was generated. If calibrated SMC information is not available the VB- SAR process can still be performed but it will not be possible to apply a depth scale to the profiles produced.

The above process provides a single depth profile from a single pixel in the input images. To obtain a VB-SAR image, this process is repeated pixel-by-pixel across the input image stack. Stacking the resulting colour-coded range profiles next to each other, forms a complete 2D VB-SAR image across the TP transect. In the case of side-looking SAR, the use of each ground pixel across the 2D scene would provide a full 3D VBSAR image.

\section{VB-SAR From High Resolution TP}

The initial test of VB-SAR was performed using the complex history of the buried trihedral extracted from the high resolution TP data. After resampling to a linear SMC trend the phase history fed to the VB-SAR processor will be a straight line. An FFT of a linear phase history will produce a single peak, at an offset from zero range defined by the gradient. For the buried trihedral, the appearance of this peak at the correct depth in the FFT output with a depth scale applied would demonstrate successful VB-SAR processing.

The result of performing VB-SAR on the phase history of the buried trihedral extracted from the high resolution TP is shown in Figure 12 below. For this process the amplitude history was artificially set to be constant at 1 to minimize any unwanted effects that might otherwise arise from the asymmetric weighting of the phase history by the brightening of the trihedral over the study period.

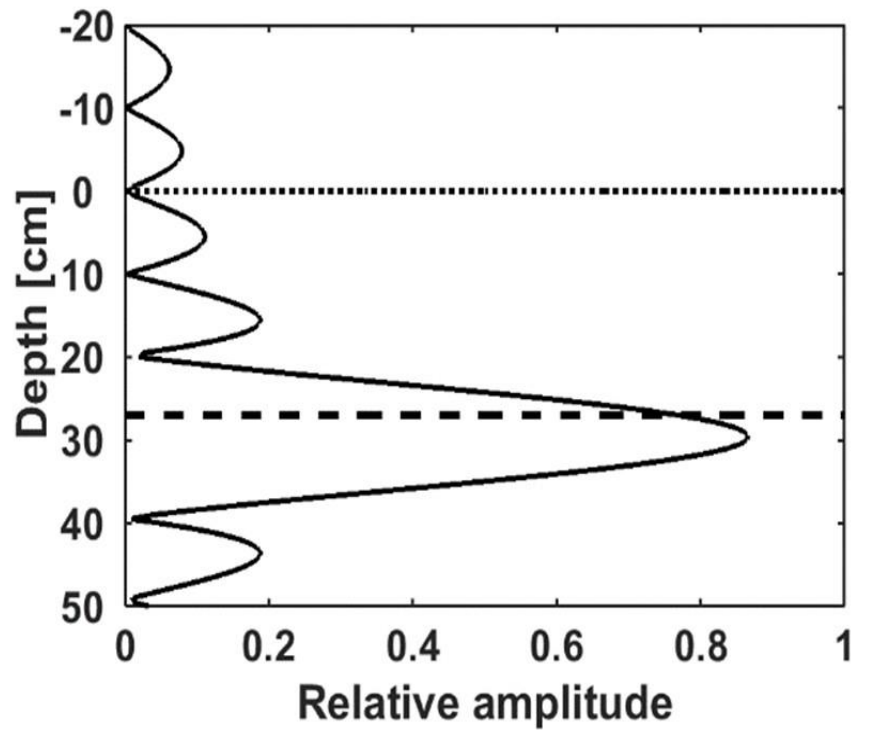

Fig. 12: VB-SAR depth profile formed using only phase history extracted from high resolution TP images. Dotted line indicates position of soil surface, dashed line indicates the position of the buried trihedral.

In order to apply an accurate depth scale, the model presented by [8] and the SMC estimates previously produced were used to calculate the refractive index of the soil in each scan. By using the average refractive index of the soil over the series of scans it was possible to calculate the virtual bandwidth. This gave the unambiguous range and hence the depth for each output bin from the VB-SAR FFT. This calculation resulted in estimates of 2.21 for the average refractive index of the soil and, from Equations (1) and (2), $3.38 \mathrm{GHz}$ for the virtual bandwidth, providing a range resolution of $0.04 \mathrm{~m}$ and an unambiguous range of $46 \mathrm{~m}$. The retrieved depth for the buried trihedral is almost $30 \mathrm{~cm}$ - close to the actual depth of $26.5 \mathrm{~cm}$ - demonstrating that the VB-SAR process is effective.

The process was repeated, but this time including the asymmetric amplitude weighting shown in Figure 10. As can be seen in Figure 13, its inclusion causes the impulse response to widen and distort, although the depth of the peak is unchanged. 


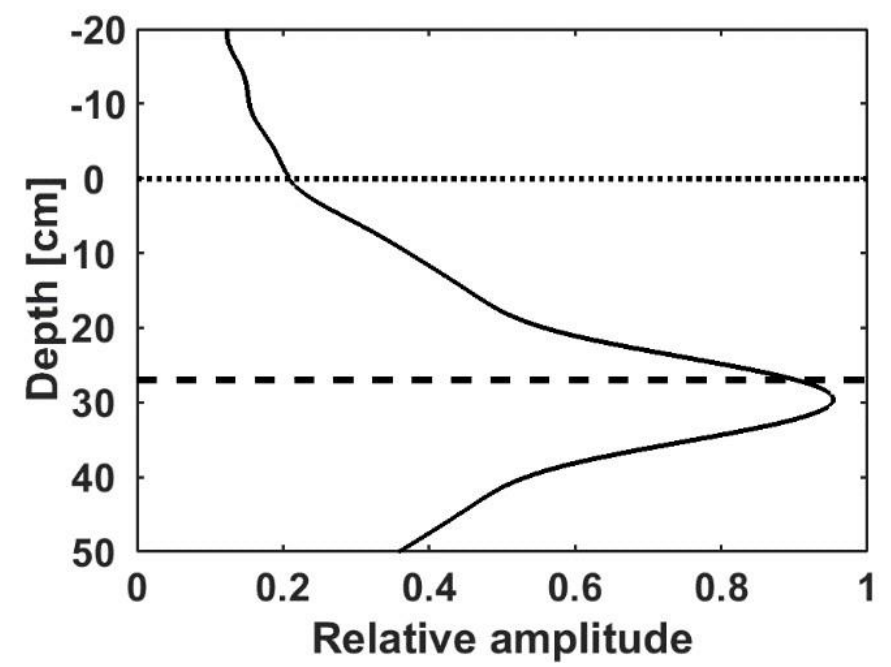

Fig. 13: VB-SAR depth profile formed using complex history extracted from high resolution TP images. Dotted line indicates position of soil surface, dashed line indicates position of buried trihedral.

\section{E. VB-SAR From Low Resolution TP}

The extraction of the buried feature's complex history is an unrealistic dataset with regard to side-looking SAR imagery gathered by satellites and aircraft. In such imagery, it would not have been possible to separate the buried trihedral returns from the overlain combination of all returns within the ground pixel. In order to emulate the data that is obtained from spaceborne side looking SAR systems with one single complex ground pixel per soil column, it is necessary to use the low resolution TP data. The lowered range resolution of $1 \mathrm{~m}$ means that a single pixel contains contributions from the soil surface, soil volume, and the buried trihedral. Application of the VBSAR process should still lead to a high resolution image product in which the buried trihedral and surface appear as separated features. Figure 14 shows an example low resolution TP image. The surface and sub-surface features can no longer be resolved, in particular, the surface and subsurface returns are no longer separable.

The VB-SAR process still requires provision of a time series in which the soil moisture changes are linear (or equivalently, the linear phase change). As such, the SMC estimates extracted from the high resolution TP data were used, which meant that the parameters calculated using the SMC estimates (average refractive index of the soil, virtual bandwidth, range resolution, unambiguous range) were identical.

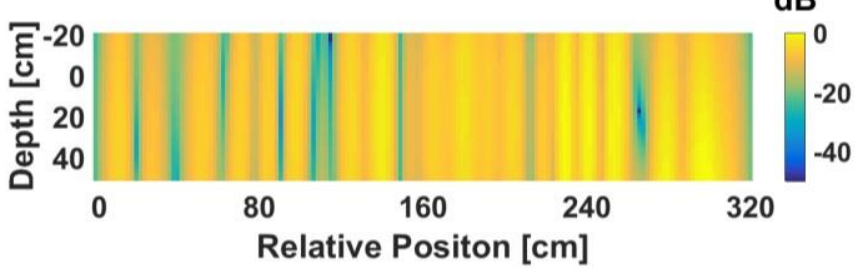

Fig. 14: Example of one of the low resolution TP images used in the VB-SAR DInSAR stack, showing that surface and sub-surfaces returns are no longer separable.
Figure 15 shows the result of applying VB-SAR to a single pixel extracted at the position of the buried trihedral across the low resolution DInSAR stack. Significantly, the surface return and the buried trihedral returns have been separated, clearly demonstrating the improvement in range resolution generated by VB-SAR processing. The buried trihedral appears at a slightly shallower depth compared to the high-resolution retrievals in Figure 12 and Figure 13, likely due to blending with the sidelobes of the surface return. This conclusion is further supported by noting that the surface return has moved fractionally deeper than $0 \mathrm{~cm}$. For Figure 16, the VB-SAR technique was applied to a region of sand that was left dry and undisturbed over the DInSAR stack. All returns, irrespective of their depth, appear at $0 \mathrm{~m}$, as the zero change in phase will be interpreted by the VB-SAR scheme as a surface target with no volume contribution. The depth scale for this depth profile was generated using the SMC history extracted from the wetted area.

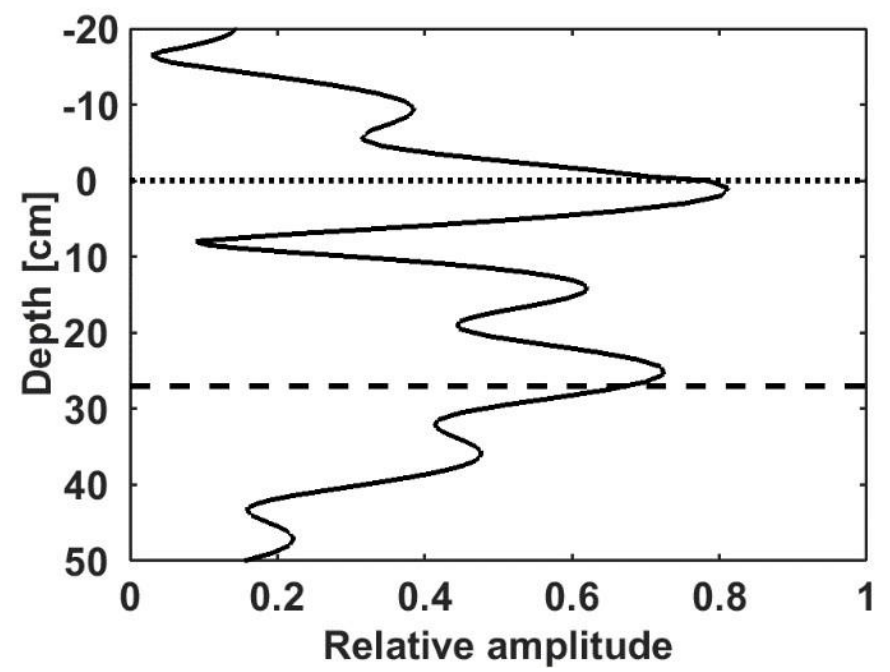

Fig. 15: VB-SAR depth profile produced from low range resolution TP data. Dotted line indicates position of soil surface, dashed line indicates position of buried trihedral.

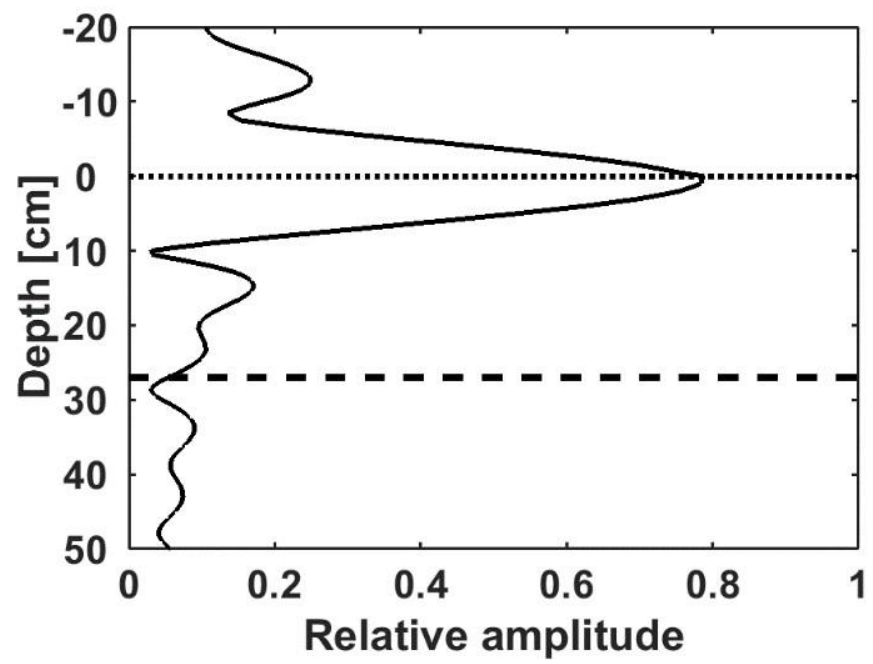

Fig. 16: VB-SAR depth profile obtained using surface pixel from dry soil area of low range resolution TP data, showing surface and all other returns correctly resolved to 0 depth. Dotted line indicates position of soil surface, dashed line indicates position of buried trihedral. 
By shortening the subaperture used during TP processing it was possible to degrade the along track resolution to $1 \mathrm{~m}$, which will have increased the clutter return (clutter being the surface and soil volume). As could be reasonably expected, the VBSAR result in Figure 17 shows that the surface return and its associated sidelobes have increased relative to the buried target's return, due to the lowered resolution primarily capturing more surface return. However, the depth of the trihedral has again been correctly recovered.

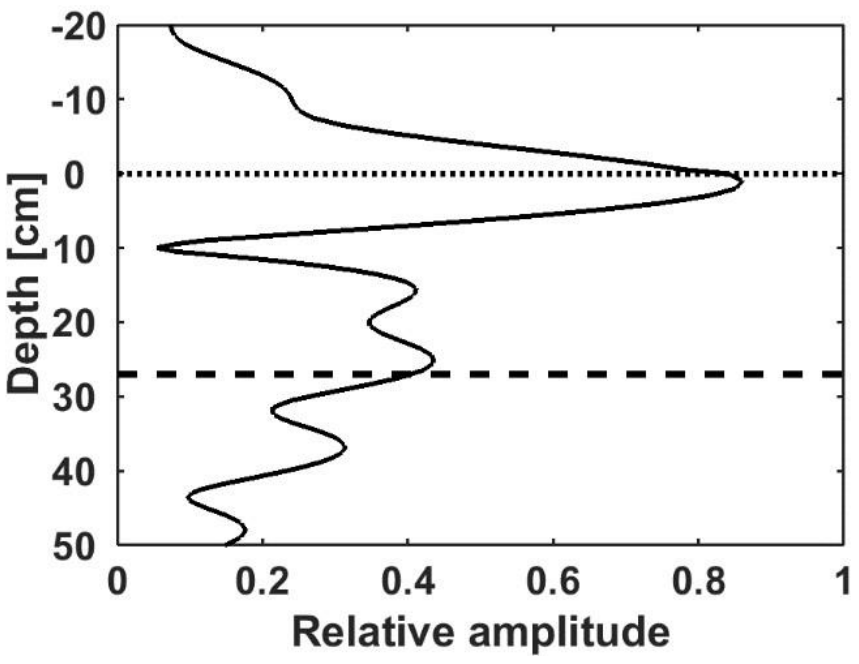

Fig. 17: VB-SAR depth profile produced from low range resolution and low cross slant range resolution TP data. Dotted line indicates position of soil surface, dashed line indicates position of buried trihedral.

As the surface should have a negligible phase change over the change in SMC [11], a direct current (DC) removal scheme should reduce the near-unchanging surface response from the depth profile. The result is shown in Figure 18, which shows that the DC removal has worked well in suppressing the surface return to improve the view of the buried feature.

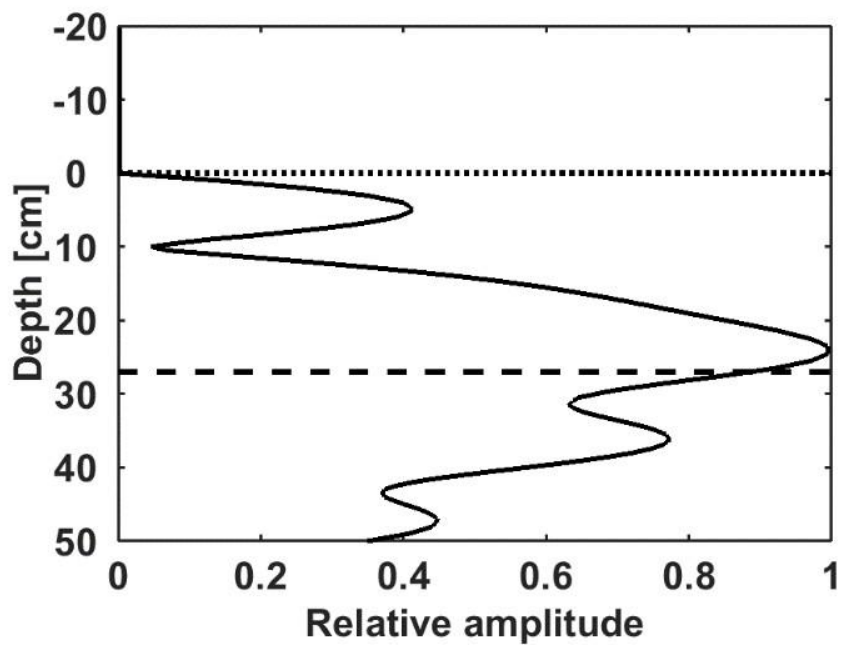

Fig. 18: VB-SAR depth profile produced from low range resolution and low cross slant range resolution TP data with DC component of complex history removed. Dotted line indicates position of soil surface, dashed line indicates position of buried trihedral.

\section{F. Full VB-SAR Imagery}

As a final demonstration of the VB-SAR scheme, a full image formed by repeating the VB-SAR process at each pixel along the trough is shown in Figure 19; a $10^{\circ}$ horizontal shear operation has been applied to the entire image in order to obtain the correct geometry. Note that the artefacts at approximately $\pm 15 \mathrm{~cm}$ depth are residual sidelobes of the strong soil surface returns that remain after the application of the Kaiser window. A heavier windowing function could further reduce these, at the expense of widening the surface and buried target peaks. The remarkable improvement the VB-SAR scheme has provided in the vertical resolution of the scene can be appreciated by comparison with Figure 14.

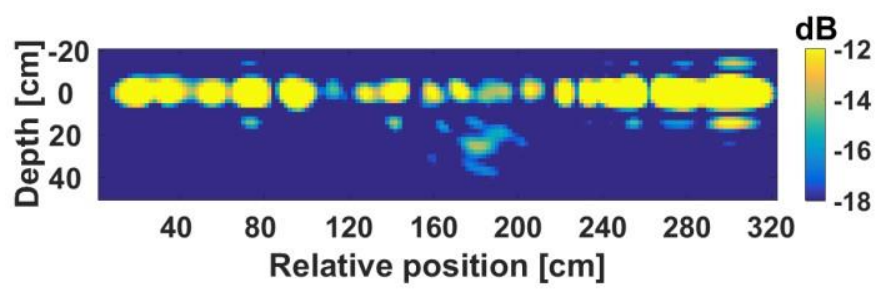

Fig. 19: Full VB-SAR image retrieved from the high resolution imagery.

In the dry areas of the scene (in this image approximately $0-110 \mathrm{~cm}$ and $220-320 \mathrm{~cm}$ horizontal position) all targets, including those buried (as indicated by arrow " $C$ " in Figure 5), appear at zero depth. This is further evidence that the VB-SAR process is working as the VB-SAR theory predicts these dry areas experienced no SMC change during the experiment so no virtual bandwidth was synthesised. Presentation of a flat phase history to the FFT results in a peak at zero depth. In these areas, all returns from all depths over the series of radar images are effectively summed and placed at zero depth which explains the very strong surface return seen in these dry areas in Figure 18.

\section{G. Real world considerations}

The application of the VB-SAR process is via interferometric processing, and production of an interferometric set would use standard DInSAR processing techniques to deal with unwanted above-ground phase terms associated with baseline separation, platform positional errors, and atmospheric moisture. The need for the maintenance of coherence between interferometric pairs means the angular separation between baselines is always small such that the fractional change in the sub-surface path (and hence phase) associated with angular differences between interferometric collections will be correspondingly small and of secondary consideration.

This study operated in a laboratory environment which allowed some simplifications and removal of likely real-world impediments. In side-looking SAR data, the phase history of any buried targets will not be isolatable from other contributions, and so the method of extracting SMC used here will not be feasible except for a dominant subsurface feature. The effect of increasing surface roughness will be to increase the surface return relative to the sub-surface return [25], likely compromising detection of the sub-surface signal.

VB-SAR separates features by virtue of differing linear phase histories, which ideally requires presentation of the phase history to the processor in equal steps of soil moisture change. Whilst in-situ measurement (either manually or with fixed automated stations) of SMC would be acceptable over small study regions, a global implementation of the scheme will require estimation of needed parameters from the SAR imagery itself. Current implementations of SMC retrieval are based on 
relationships between moisture and backscatter brightness, and these could be utilised for the estimation of moisture parameters for use by the VB-scheme [13-16]. If SMC information is not available, it is possible to operate the VB-SAR scheme in a "detection mode". This would omit the SMC linearisation step and pass the phase history directly to the FFT. For moderate phase non-linearity subsurface objects would still show a phase shift and so appear separated and below the surface, although the depth scale would be inaccurate [9]. In a case of extreme divergence from linearity, the phase disturbance evident at a pixel would indicate the presence of a buried feature but without being able to produce a properly focused image or correct depth scale. For a sloping or uneven surface, a sub-surface feature will appear at a representative "effective depth" just as is the case for an interferometric height retrieval across an undulating forest canopy. The VB-SAR scheme only improves the below ground slant range resolution; cross slant range resolution is unimproved so objects at the same depth in a ground pixel will appear as one combined return in the VB-SAR depth pixel.

There is a question of whether subsurface objects would be detectable using existing radar platforms. Current spaceborne SAR systems have Noise Equivalent Sigma Zero (NESZ) figures on the order of -20dB [17-19], such that even modest attenuations associated with low SMC will rapidly compromise measurement of subsurface features. In comparison, airborne systems typically have an extra $30 \mathrm{~dB}$ of sensitivity and so are currently more suited to VB-SAR [20-22]. Geologic and climatic considerations produce preferred regions for VB-SAR; a soil should be as dry as possible to minimise attenuation whilst still providing a large enough change in SMC to generate sufficient virtual bandwidths. This tradeoff will vary for different targets and different radar systems but it can be modelled using the soil model found in [8].

\section{CONCLUSIONS}

This study has demonstrated VB-SAR imaging within the laboratory environment. A scene consisting of a buried trihedral under a sand and gravel mixture was set up and the scene repeatedly imaged. Controlled amounts of water were then added to the surface of the sand and gravel mixture, and the scene was allowed to dry naturally which produced a radar data set covering both wetting and drying periods. The imagery was fed to the VB-SAR processor which successfully produced high-resolution subsurface imagery for two different cases; firstly using the complex history of a buried trihedral only; and secondly, using the combined complex histories that contained contributions from the surface, soil volume and a buried trihedral. The latter case better emulated the situation with data from a real world, side-looking SAR system.

The study has validated the basic concept of VB-SAR; it has successfully demonstrated stand-off subsurface imaging with a vastly increased range resolution over that given by the real bandwidth of the radar system, and provided direct and unambiguous discrimination of surface and subsurface objects. Techniques for mitigating complications that a real world VBSAR scheme may have to contend with were also discussed. Future experimental work will include polarimetricdependencies of VB-SAR.

\section{ACKNOWLEDGMENTS}

The authors are grateful to Cranfield University for supporting this work through an internal bursary award, Dr. Mark Finnis for extensive assistance with the GB-SAR system and Dr. Daniel Andre for useful technical discussions.

\section{REFERENCES}

[1] D. J. Daniels, Ground Penetrating Radar, Encyclopaedia of RF and Microwave Engineering, John Wiley \& Sons, 2005.

[2] A.G. Yarovoy, T.G Savelyev, P.J. Aubry, and L.P. Ligthart, "Array-Based GPR for Shallow Subsurface Imaging," Advanced Ground Penetrating Radar, 2007 4th Int. Workshop on , pp. 12-15, June 2007.

[3] M. Sato, K. Doi, and K. Takahashi, "Advanced GPR for archaeological survey," Geosci. and Remote Sensing Symp. (IGARSS), 2013 IEEE Int., pp. 1395-1398, July 2013.

[4] T. G. Farr and P. Paillou, "Radar Remote Sensing of the Sahara Landscape." Proc. 9th European Conf. on Synthetic Aperture Radar, pp. 717-718, April 2012.

[5] P. Paillou and A. Rosenqvist, "A JERS-1 radar mosaic for subsurface geology mapping in East Sahara," Geosci. and Remote Sensing Symp. (IGARSS), 2003 IEEE Int., vol. 3, pp. 1870-1872, July 2003.

[6] P. Paillou, S. Lopez, T. Farr, and A. Rosenqvist, "Mapping Subsurface Geology in Sahara Using L-Band SAR: First Results From the ALOS/PALSAR Imaging Radar." IEEE J. Sel. Topics Appl. Earth Observ. in Remote Sens., vol. 3 no. 4, pp. 632-636, 2010.

[7] J. Mccauley, G. Schaber, C. Breed, M. Grolier, C. Haynes, B. Issawi, C. Elachi, and R. Blom, "Subsurface valleys and geoarcheology of the eastern Sahara revealed by shuttle radar," Science, vol. 218, no. 4576, pp. 1004-1020, 1982.

[8] M. Hallikainen, F. Ulabz, M. Dobson, M. El-Rayes, and L.K. Wu, "Microwave dielectric behavior of wet soil-part I: Empirical models and experimental observations," IEEE Trans. on Geosci. and Remote Sens., vol. GE-23, no. 1, pp. 25-34, 1985.

[9] K. Morrison and J. Bennett, "Virtual bandwidth SAR (VB-SAR) for centimetric-scale sub-surface imaging from space," Int. J. Remote Sensing, vol. 36, no. 7, pp. 1789-1808, 2015.

[10] K. Morrison and J. Bennett, "Tomographic profiling - a technique for multi-incidence-angle retrieval of the vertical SAR backscattering profiles of biogeophysical targets," IEEE Trans. on Geosci. and Remote Sens., vol. 52, no. 2, pp. 1250-1255, 2014.

[11] K. Morrison, J.C. Bennett, and M. Nolan, "Using DInSAR to Separate Surface and Subsurface Features," IEEE Trans. on Geosci. and Remote Sens., vol. 51, no. 6, pp. 3424-3430, 2013.

[12] V. Mironov Dobson, V. H. Kaupp, S. Komarov, and V. Kleshchenko, "Generalized refractive mixing dielectric model for moist soils," IEEE Trans. on Geosci. and Remote Sens., vol. 42, no. 4, pp.773-785, 2004. 
[13] P. Dubois, J. Van Zyl, and T. Engman, "Measuring soil moisture with imaging radars," IEEE Trans. on Geosci. and Remote Sens., vol. 33, no. 4, pp. 915-926, 1995.

[14] P. J. van Oevelen and D. H. Hoekman, "Radar backscatter inversion techniques for estimation of surface soil moisture: EFEDA-Spain and HAPEX-Sahel case studies," IEEE Trans. on Geosci. and Remote Sens., vol. 37, no. 1, pp. 113-123, 1999.

[15] M. Zribi, N. Baghdadi, N. Holah, and O. Fafin, "New methodology for soil surface moisture estimation and its application to ENVISAT-ASAR multi-incidence data inversion," Remote Sensing of Environment, 96 (3-4), pp. 485-496, 2005.

[16] N.G. Meade, L.D. Hinzman, and D.L. Kane, "Spatial estimation of soil moisture using synthetic aperture radar in Alaska", Advances in Space Research, Volume 24, Issue 7, Pages 935-940, 1999.

[17] G. Krieger, A. Moreira, H. Fiedler, I. Hajnsek, M. Eineder, M. Zink, and M. Werner, "TanDEM-X: A Satellite Formation for High-Resolution SAR Interferometry," IEEE Trans. on Geosci. and Remote Sens., vol. 45, no. 11, pp. 3317-3341, 2007.

[18] “Sentinel-1: ESA's Radar Observatory Mission for GMES Operational Services” ESA SP 1322/1, March 2012.

[19] Y. Kankaku, M. Sagisaka, and S. Suzuki, "PALSAR-2 launch and early orbit status," Geosci. and Remote Sensing Symp. (IGARSS), 2014 IEEE Int., Quebec City, QC, pp. 3410-3412, July 2014.

[20] A. G. Fore, B.D. Chapman, B.P. Hawkins, S. Hensley, C.E. Jones, T.R. Michel, and R.J. Muellerschoen, "UAVSAR Polarimetric Calibration," IEEE Trans. on Geoscience and Remote Sens., vol. 53, no. 6, pp. 34813491, 2015.

[21] A. Reigber, M. Jäger, J. Fischer, R. Horn, R. Scheiber, P. Prats, and A. Nottensteiner, "System status and calibration of the F-SAR airborne SAR instrument," Geosci. and Remote Sensing Symp. (IGARSS), 2011 IEEE Int., Vancouver, BC, pp. 1520-1523, July 2011.

[22] R. Baque, R. Baqué, and P. Dreuillet, "The airborne SARsystem: RAMSES NG airborne microwave remote sensing imaging system," Radar Syst. (Radar 2012), IET Int. Conf. on, Glasgow, UK, pp. 1-4, October 2012.

[23] S. Zwieback, S. Hensley, I. Hajnsek, "A polarimetric firstorder model of soil moisture effects on the DInSAR coherence", Remote Sensing, vol. 7, no. 6, pp. 7571-7596. 2015.

[24] A. Moreira, P. Prats-Iraola, M. Younis, G. Krieger, I. Hajnsek and K. P. Papathanassiou, "A tutorial on synthetic aperture radar", IEEE Geoscience and Remote Sensing Magazine, vol. 1, no. 1, pp. 6-43, March 2013.

[25] J. Shi, J. Wang, A. Y. Hsu, P. E. O'Neill, and E. T. Engman, "Estimation of bare surface soil moisture and surface roughness parameter using L-band SAR image data," IEEE Trans. on Geosci. and Remote Sens., vol. 35, no. 5, pp. 1254-1266,1997. 